PROCEEDINGS OF THE

AMERICAN MATHEMATICAL SOCIETY

Volume 138, Number 3, March 2010, Pages 975-985

S 0002-9939(09)10181-8

Article electronically published on October 26, 2009

\title{
GLOBAL STABILITY OF A CLASS OF NON-AUTONOMOUS DELAY DIFFERENTIAL SYSTEMS
}

\author{
BINGWEN LIU
}

(Communicated by Yingfei Yi)

\begin{abstract}
This paper is concerned with a class of systems of non-autonomous delay differential equations which are defined on the non-negative function space. Under proper conditions, we employ a novel proof to establish several criteria of the global stability of a positive equilibrium. Moreover, we give two examples to illustrate our main results.
\end{abstract}

\section{INTRODUCTION}

In this paper, we consider the following system of delay differential equations:

$$
x^{\prime}(t)=f\left(t, x_{t}\right)
$$

where $f: R^{1} \times \prod_{i=1}^{n} C\left(\left[-r_{i}, 0\right], R^{1}\right) \rightarrow R^{n}$ is completely continuous, $f(t, \varphi)$ is locally Lipschitzian in $\varphi, f(t, \widehat{0}) \equiv f\left(t, \widehat{x^{*}}\right) \equiv 0$ with $x^{*} \triangleq\left(x_{1}^{*}, x_{2}^{*}, \cdots, x_{n}^{*}\right) \in I_{n} R_{+}^{n}$, and $r_{i}$ is a positive constant for $i=1,2, \cdots, n$.

To further our discussion, we also assume that the functional $f(t, \varphi)$ is almost periodic in $t$ for fixed $\varphi \in \prod_{i=1}^{n} C\left(\left[-r_{i}, 0\right], R^{1}\right)$.

When $n=1$, system (1.1) is a class of general scalar delay differential equations, including the Nicholson blowflies equation and the logistic-type delay equation. Recently, the Nicholson blowflies equation and the logistic-type delay equation have served as the models for some population dynamics and ecology problems which have been extensively studied by many authors, and many interesting results have been obtained; see for example [1, 4, 7, 8. Here we shall investigate the global stability of the positive equilibrium of (1.1). For the system with a large $n$, several methods have been used, such as the Liapunov functional method, the invariance principle of Liapunov-Rarumikhin type and the monotone method, to investigate the global dynamics of (1.1) (see [2, 3, 5, 6, 9]). Moreover, in the application of the Liapunov functional method, the authors often require that the Liapunov functional's derivative along (1.1) is less than or equal to 0 (see [3] and the references cited therein). Before illustrating our study method, we shall recall one

Received by the editors June 25, 2009.

2010 Mathematics Subject Classification. Primary 34D05, 34C11, 34C12.

Key words and phrases. Delay differential equation, non-autonomous, global stability, nonnegative function space, positive equilibrium.

This work was supported by the National Natural Science Foundation of PR China (Grants No. 10801047, 10971229).

(C)2009 American Mathematical Society Reverts to public domain 28 years from publication 
recent paper [8] on the global attractivity of the positive steady state of the diffusive Nicholson equation. By combining a dynamical systems argument with the maximum principle and some subtle inequalities, the authors of [8] have obtained the global attractivity of the positive steady state of the diffusive Nicholson equation with homogeneous Neumann boundary value under a condition that makes the equation a non-monotone dynamical system. This synthetic method motivates us to integrate the Liapunov functional with other methods to analyze the global stability of the positive equilibrium of (1.1).

In fact, we first construct a "proper" Liapunov functional under some appropriate conditions. However, we usually cannot deduce the Liapunov functional's derivatives along (1.1). Therefore, some alternative methods are required. Then, by the contrary argument and the comparison technique, we show that the Liapunov functional along every non-trivial solution of (1.1) is either strictly decreasing or eventually 0. Finally, by applying the basic theory of the Liapunov direct method and the properties of the Liapunov functional, we obtain the global stability of (1.1).

As some applications, we also consider the following non-autonomous Nicholson blowflies equation with patch structure:

$$
x_{i}^{\prime}(t)=\sum_{j=1}^{n} a_{i j} x_{j}(t)+\beta x_{i}\left(t-\tau_{i}(t)\right) e^{-x_{i}\left(t-\tau_{i}(t)\right)}-d x_{i}(t), i=1,2, \cdots, n,
$$

and non-autonomous delay logistic equation with patch structure:

$$
x_{i}^{\prime}(t)=\sum_{j=1}^{n} a_{i j} x_{j}(t)+k_{i} x_{i}(t)\left[\alpha_{i}-b_{i 0} x_{i}(t)-\sum_{j=1}^{m} b_{i j} x_{i}\left(t-\tau_{j}(t)\right)\right], i=1,2, \cdots, n .
$$

The remaining part of this paper is organized as follows. In Section 2 we give some basic definitions and preliminary results. In Section 3, by applying the Liapunov direct method, we establish several criteria of the global stability of (1.1). In Section 4, we make some applications to the non-autonomous Nicholson blowflies equation and/or non-autonomous logistic equation with patch structure.

\section{Definitions AND PRELIMINARY RESUlts}

In this paper, we denote by $R^{n}\left(R_{+}^{n}\right)$ the set of all (non-negative) real vectors. We will use $x=\left(x_{1}, x_{2}, \cdots, x_{n}\right) \in R^{n}$ to denote a column vector and define $|x|=\max _{1 \leq i \leq n}\left|x_{i}\right|$. Let $\left(r_{1}, r_{2}, \cdots, r_{n}\right) \in \operatorname{Int} R_{+}^{n}$ be given and $C=\prod_{i=1}^{n} C\left(\left[-r_{i}, 0\right], R^{1}\right)$ be the continuous functions space equipped with the usual supremun norm $\|\cdot\|$. We also let

$$
r=\max _{1 \leq i \leq n} r_{i}, I=\{1,2, \cdots, n\}, C_{+}=\prod_{i=1}^{n} C\left(\left[-r_{i}, 0\right], R_{+}^{1}\right), C_{H}=\{\varphi \in C:\|\varphi\| \leq H\},
$$

and

$$
C_{H(L)}=\left\{\varphi \in C_{H}:\left|\varphi_{i}\left(t_{1}\right)-\varphi_{i}\left(t_{2}\right)\right| \leq L\left|t_{1}-t_{2}\right| \text { for each } t_{1}, t_{2} \in\left[-r_{i}, 0\right], i \in I\right\},
$$

where $H>0$ and $L>0$ are constants.

If $x_{i}(t)$ is defined on $\left[t_{0}-r_{i}, \sigma\right)$ with $t_{0}, \sigma \in R^{1}$ and $i \in I$, then we define $x_{t} \in C$ as $x_{t}=\left(x_{t}^{1}, x_{t}^{2}, \cdots, x_{t}^{n}\right)$, where $x_{t}^{i}(\theta)=x_{i}(t+\theta)$ for all $\theta \in\left[-r_{i}, 0\right]$ and $i \in I$. For 
any $\varphi, \psi \in C$, we write $\varphi \leq \psi$ if $\psi-\varphi \in C_{+}, \varphi<\psi$ if $\varphi \leq \psi$ and $\varphi \neq \psi$. For any $x, y \in R^{n}$, we write $x \leq y$ if $x-y \in R_{+}^{n}, x<y$ if $x \leq y$ and $x \neq y$. Similarly, we can define " $\geq$ " and " $>$ ". For $x=\left(x_{1}, x_{2}, \cdots, x_{n}\right) \in R^{n}$, we write $\widehat{x}$ for the element of $C$ satisfying $\widehat{x}=\left(\widehat{x_{1}}, \widehat{x_{2}}, \cdots, \widehat{x_{n}}\right)$, where $\left(\widehat{x_{i}}\right)\left(\theta_{i}\right)=x_{i}$ for all $\theta_{i} \in\left[-r_{i}, 0\right]$ and $i \in I$.

We write $x_{t}\left(t_{0}, \varphi\right)\left(x\left(t ; t_{0}, \varphi\right)\right)$ for a solution of the initial value problem (1.1) with $x_{t_{0}}\left(t_{0}, \varphi\right)=\varphi \in C$ and $t_{0} \in R^{1}$. Also, let $\left[t_{0}, \eta(\varphi)\right)$ be the maximal right-interval of existence of $x_{t}\left(t_{0}, \varphi\right)$.

Definition 2.1 (3). A continuous function $F(t): R^{1} \rightarrow R^{n}$ is called almost periodic if for every $\varepsilon>0$ there exists $l=l(\varepsilon)>0$ such that any segment $[\alpha, \alpha+l], \alpha \in R^{1}$, contains at least one number $\tau$ such that $|F(t+\tau)-F(t)|<\varepsilon$ for every $t \in R^{1}$. A number $\tau$ is called an $\varepsilon$-almost period of $F$.

Definition 2.2 (3). A continuous functional $F(t, \varphi): R^{1} \times C_{H(L)} \rightarrow R^{n}$ is called uniformly almost periodic in $t$ if for every $\varepsilon>0$ there exists $l=l(\varepsilon)>0$ such that any segment $[\alpha, \alpha+l], \alpha \in R^{1}$, contains at least one number $\tau$ such that $|F(t+\tau, \varphi)-F(t, \varphi)|<\varepsilon$ for all $t \in R^{1}, \varphi \in C_{H(L)}$.

Lemma 2.1 (3). Let $F_{1}(t), \cdots, F_{N}(t): R^{1} \rightarrow R^{n}$ be almost periodic functions. Then for every $\varepsilon>0$ there exists $l=l(\varepsilon)>0$ such that any segment $[\alpha, \alpha+l], \alpha \in$ $R^{1}$, contains a number $\tau$ such that

$$
\left|F_{i}(t+\tau)-F_{i}(t)\right|<\varepsilon, i=1,2, \cdots, N, t \in R^{1} .
$$

Lemma 2.2. If $L$ is a positive constant and if the functional $F(t, \varphi): R^{1} \times C_{H(L)} \rightarrow$ $R^{n}$ is Lipschitzian in $\varphi$ and almost periodic in $t$ for every fixed $\varphi \in C_{H(L)}$, then it is uniformly almost periodic in $t$.

Proof. Since the functional $F(t, \varphi)$ satisfies Lipschitz conditions in $\varphi$, then there exists a constant $L_{1}>0$ such that

$$
|F(t, \varphi)-F(t, \varphi)| \leq L_{1}\|\varphi-\psi\|, \text { for all } \varphi, \psi \in C_{H(L)} .
$$

Let $\varepsilon>0$ be any real number. From the definition of $C_{H(L)}$ and the Ascoli-Arzelà Theorem, we have that $C_{H(L)}$ is a compact set. Hence there exist $\varphi^{1}, \varphi^{2}, \cdots, \varphi^{N}$ $\in C_{H(L)}$ such that

$$
\min _{i \in I_{N}}\left\|\varphi-\varphi^{i}\right\|<\frac{\varepsilon}{3 L_{1}} \text { for all } \varphi \in C_{H(L)},
$$

where $I_{N} \triangleq\{1,2, \cdots, N\}$. By Lemma 2.1, it follows that there exists $l>0$ such that in any segment $[\alpha, \alpha+l]$ there exists a number $\tau$ such that

$$
\left|F\left(t, \varphi^{i}\right)-F\left(t+\tau, \varphi^{i}\right)\right|<\frac{\varepsilon}{3} \text { for all } t \in R^{1}, i \in I_{N} .
$$

Then, for every $\varphi \in C_{H(L)}$, there exists $k \in I_{N}$ such that

$$
\left\|\varphi-\varphi^{k}\right\|<\varepsilon / 3 L_{1}
$$

Thus, from $(2.4)-(2.6)$, we obtain

$$
\begin{aligned}
& |F(t+\tau, \varphi)-F(t, \varphi)| \leq\left|F(t+\tau, \varphi)-F\left(t+\tau, \varphi^{k}\right)\right| \\
& +\left|F\left(t+\tau, \varphi^{k}\right)-F\left(t, \varphi^{k}\right)\right|+\left|F\left(t, \varphi^{k}\right)-F(t, \varphi)\right| \\
& <\frac{\varepsilon}{3}+2 L_{1} \cdot \frac{\varepsilon}{3 L_{1}}=\varepsilon .
\end{aligned}
$$

The inequality (2.7) implies Lemma 2.2. 
Remark 2.1. By locally Lipschitzian conditions and the compactness of $C_{H(L)}$, we obtain that the functional $f(t, \varphi)$ is Lipschitzian in $C_{H(L)}$. Again by Lemma 2.2, $F(t, \varphi)=\left.f(t, \varphi)\right|_{R \times C_{H(L)}}$ is uniformly almost periodic in $t$.

Lemma 2.3 (3. Lemma 3]). For a fixed $\varphi^{0} \in C$, we suppose that $x_{t}\left(t_{0}, \varphi^{0}\right)$ belongs to $C_{H}$ for all $t \geq t_{0}$. Then there exist constants $L>0$ and $r^{*}>r$ such that

(i) $x_{t}\left(t_{0} ; \varphi^{0}\right) \in C_{H(L)}$ for all $t \geq t_{0}+r^{*}$.

(ii) Let $\left\{\varepsilon_{k}\right\}_{k=1}^{+\infty}$ be a monotonically approaching zero sequence of positive numbers and $\left\{\tau_{k}: \tau_{k}>r^{*}\right\}_{k=1}^{+\infty}$ be a sequence of $\varepsilon_{k}$-almost periods of $f(t, \varphi)$ for all $\varphi \in C_{H(L)}$ (for every $\varepsilon_{k}$ there corresponds an $\varepsilon_{k}$-almost period $\tau_{k}$ ). Then, for a given $t^{*}>t_{0}$, we get

$$
\lim _{k \rightarrow+\infty}\left\|x_{t^{*}}\left(t_{0}, \varphi^{k}\right)-x_{t^{*}+\tau_{k}}\left(t_{0}, \varphi^{0}\right)\right\|=0
$$

with $\varphi^{k}=x_{t_{0}+\tau_{k}}\left(t_{0}, \varphi^{0}\right)$.

Remark 2.2. From Definition 2.1, we can choose a monotonically approaching $+\infty$ sequence $\left\{\tau_{k}: \tau_{k}>r^{*}\right\}_{k=1}^{+\infty}$ satisfying Lemma 2.3.

\section{MAin RESUlts}

We first introduce the following proposition:

Proposition 3.1. Suppose that the system (1.1) has a unique solution $x_{t}\left(t_{0}, \varphi\right)$ on $\left[t_{0},+\infty\right)$ with $x_{t_{0}}\left(t_{0}, \varphi\right)=\varphi \in C_{+}$. We also assume that there exists a positive constant $m$ such that

$$
\left\|x_{t}\left(t_{0}, \varphi\right)\right\| \leq\|\varphi\|+m\left\|x^{*}\right\| \text { for all } s \geq t_{0}, \varphi \in C_{+} .
$$

Let $V(\varphi)=\left\|\varphi-x^{*}\right\| \triangleq \sup \left\{\left|\varphi_{i}(\theta)-x_{i}^{*}\right|: i \in I\right.$ and $\left.\theta \in\left[-r_{i}, 0\right]\right\}$ for all $\varphi \in C_{+}$. Moreover, there exist $T_{1} \geq 0$ and $M \subseteq\left\{\varphi \in C_{+}: \varphi(0)=0\right\}$ with $0 \in M$ such that the following conditions hold:

(i) $x_{t}\left(t_{0}, \varphi\right) \in \operatorname{Int}\left(C_{+}\right)$for $\varphi \in C_{+} \backslash M$ and $t \geq T_{1}+t_{0}$.

(ii) If $\varphi \in C_{+} \backslash M$ and $x_{t}\left(t_{0}, \varphi\right) \neq x^{*}$ for all $t \in\left[t_{0},+\infty\right)$, then $V\left(x_{t}\left(t_{0}, \varphi\right)\right)$ is strictly decreasing on $\left[T_{1}+t_{0},+\infty\right)$.

(iii) $\varphi \in C_{+} \backslash M$ and $V\left(x_{t}\left(t_{0}, \varphi\right)\right)$ is non-increasing on $\left[T_{1}+t_{0},+\infty\right)$.

Then $x^{*}$ is a globally stable equilibrium on $C_{+} \backslash M$.

Proof. We now prove that $x^{*}$ is a stable equilibrium. For any $\epsilon>0$, it follows from the continuous dependence of the solution of (1.1) that there is $\delta \in$ $\left[0, \min \left\{\epsilon, \min \left\{x_{i}^{*}: i \in I\right\}\right\}\right)$ such that $V\left(x_{t}\left(t_{0}, \varphi\right)\right)<\epsilon$ for all $t \in\left[t_{0}, T_{1}+t_{0}\right]$ and $\varphi \in C_{+} \backslash M$ with $V(\varphi)<\delta$. On the other hand, the assumption (iii) implies $V\left(x_{t}\left(t_{0}, \varphi\right)\right) \leq V\left(x_{T_{1}+t_{0}}\left(t_{0}, \varphi\right)\right)<\epsilon$ for all $t \in\left[T_{1}+t_{0},+\infty\right)$ and $\varphi$ with $V(\varphi)<\delta$. Hence, $V\left(x_{t}\left(t_{0}, \varphi\right)\right)<\epsilon$ for all $t \in\left[t_{0},+\infty\right)$ and $\varphi$ with $V(\varphi)<\delta$. So, $x^{*}$ is a stable equilibrium.

We next show that $x^{*}$ attracts $C_{+} \backslash M$. Given $\varphi \in C_{+} \backslash M$, from (iii), we have that $\lim _{t \rightarrow+\infty} V\left(x_{t}\left(t_{0}, \varphi\right)\right)=V_{0}$ for some $V_{0} \geq 0$. We shall show $V_{0}=0$; otherwise, $V_{0}>0$, and it is easy to see that $V\left(x_{t}\left(t_{0}, \varphi\right)\right) \geq V_{0}$ for all $t \in\left[T_{1}+t_{0},+\infty\right)$.

Set $H=\|\varphi\|+2 m\left\|x^{*}\right\|$. Then, from (3.1), we have

$$
x_{t}\left(t_{0}, \varphi\right) \in C_{H} \text { for all } t \geq t_{0} .
$$

By Lemma 2.3, we can choose positive constants $L$ and $r^{*}>r$ such that

$$
x_{t}\left(t_{0}, \varphi\right) \in C_{H(L)} \text { for all } t \geq t_{0}+r^{*} .
$$


By Remark 2.2, let $\left\{\varepsilon_{k}\right\}_{k=1}^{+\infty}$ be a monotonic sequence of positive numbers and $\left\{\tau_{k}: \tau_{k} \geq r^{*}\right\}_{k=1}^{+\infty}$ be a monotonic sequence of $\varepsilon_{k}$-almost periods of $f(t, \varphi)$ for all $\varphi \in C_{H(L)}$ (for every $\varepsilon_{k}$ there corresponds an $\varepsilon_{k}$-almost period $\tau_{k}$ ) such that $\lim _{k \rightarrow+\infty} \varepsilon_{k}=0$ and $\lim _{k \rightarrow+\infty} \tau_{k}=+\infty$. Then, again by Lemma 2.3, for a given $t^{*} \geq t_{0}$, we get

$$
\lim _{k \rightarrow+\infty}\left\|x_{t^{*}}\left(t_{0}, \varphi^{k}\right)-x_{t^{*}+\tau_{k}}\left(t_{0}, \varphi\right)\right\|=0
$$

with $\varphi^{k}=x_{t_{0}+\tau_{k}}(\varphi)$.

Without loss of generality, we may assume that

$$
\lim _{k \rightarrow+\infty} \varphi^{k}=\varphi^{*} \text { for some } \varphi^{*} \in C_{+} .
$$

From (3.3) and the continuity of $V(\cdot)$ and $x_{t}\left(t_{0}, \cdot\right)$, we obtain

$$
V\left(x_{t}\left(t_{0}, \varphi^{*}\right)\right)=\lim _{k \rightarrow+\infty} V\left(x_{t}\left(t_{0}, \varphi^{k}\right)\right)=\lim _{k \rightarrow+\infty} V\left(x_{t+\tau_{k}}\left(t_{0}, \varphi\right)\right)=V_{0} \text { for all } t \geq t_{0} .
$$

We shall prove that

$$
x_{t_{1}}\left(t_{0}, \varphi^{*}\right) \notin M \text { for some } t_{1} \in\left[t_{0}, t_{0}+r\right] .
$$

Otherwise,

$$
x_{t_{0}+r}\left(t_{0}, \varphi^{*}\right)=0 \text { and } V_{0}=\left\|x^{*}\right\|,
$$

which, together with (3.3), imply that

$$
\lim _{k \rightarrow+\infty}\left\|x_{t_{0}+r+\tau_{k}}\left(t_{0}, \varphi\right)\right\|=0 .
$$

On the other hand, by the assumptions $(i),(i i i)$ and $\varphi \in C_{+} \backslash M$, we have

$$
x_{t}\left(t_{0}, \varphi\right) \in \operatorname{Int}\left(C_{+}\right) \text {for all } t \geq T_{1}+t_{0}
$$

and

$$
V\left(x_{t}\left(t_{0}, \varphi\right)\right) \text { is non-increasing on }\left[T_{1}+t_{0},+\infty\right) .
$$

Obviously, there exists a positive integer $k_{0}$ such that the following statements hold:

(1) $x_{t_{0}+r+\tau_{k}}\left(t_{0}, \varphi\right) \in \operatorname{Int}\left(C_{+}\right)$for $k \geq k_{0}$;

(2) $V\left(x_{t_{0}+r+\tau_{k}}\left(t_{0}, \varphi\right)\right)$ is non-increasing for $k \geq k_{0}$;

(3) $x_{t_{0}+r+\tau_{k}}\left(t_{0}, \varphi\right) \in C_{H^{*}}$ for $k \geq k_{0}$, where $H^{*}=\min _{i \in I}\left\{\frac{x_{i}^{*}}{5}\right\}$.

It follows from (1), (3) and the definition of $V(\cdot)$ that

$$
\begin{aligned}
V\left(x_{t_{0}+r+\tau_{k}}\left(t_{0}, \varphi\right)\right) & =\sup \left\{x_{i}^{*}-x_{i}\left(t_{0}+r+\tau_{k}+\theta_{i} ; t_{0}, \varphi\right): i \in I \text { and } \theta_{i} \in\left[-r_{i}, 0\right]\right\} \\
& <\left\|x^{*}\right\| \text { for all } k \geq k_{0} .
\end{aligned}
$$

This and (2) imply that

$$
V\left(x_{t_{0}+r+\tau_{k}}\left(t_{0}, \varphi\right)\right) \leq V\left(x_{t_{0}+r+\tau_{k_{0}}}\left(t_{0}, \varphi\right)\right)<\left\|x^{*}\right\| \text { for all } k \geq k_{0} .
$$

Hence, by (2) and (3.4), we obtain $\lim _{k \rightarrow+\infty} V\left(x_{t_{0}+r+\tau_{k}}\left(t_{0}, \varphi\right)\right)<\left\|x^{*}\right\|$, a contradiction to $V_{0}=\left\|x^{*}\right\|$.

So, there exists $t_{1} \in\left[t_{0}, t_{0}+r\right]$ such that

$$
x_{t_{1}}\left(t_{0}, \varphi^{*}\right) \in C_{+} \backslash M .
$$

Let $\varphi^{* *}=x_{t_{1}}\left(t_{0}, \varphi^{*}\right)$. Then,

$$
\varphi^{* *} \in C_{+} \backslash M \text { and } x_{t}\left(t_{0}, \varphi^{*}\right)=x_{t}\left(t_{1}, x_{t_{1}}\left(t_{0}, \varphi^{*}\right)\right)=x_{t}\left(t_{1}, \varphi^{* *}\right) \text { for all } t \geq t_{1} \text {. }
$$

By applying the conditions $(i)-($ iii $)$ of Proposition 3.1 to $\varphi^{* *}$, we have

$$
V\left(x_{t}\left(t_{0}, \varphi^{*}\right)\right) \text { is strictly decreasing for all } t \geq T_{1}+t_{1} \text {, }
$$


a contradiction to (3.4). This completes the proof Proposition 3.1.

In the remainder of this paper, we shall introduce the following assumptions to guarantee the global stability of system (1.1):

(A1) If $\varphi \in C_{+}$and $t \in\left[t_{0}, \eta(\varphi)\right)$, then $x_{t}\left(t_{0}, \varphi\right) \in C_{+}$.

(A2) If $\varphi \in C_{+} \backslash\{0\}$ and $t \in\left[t_{0}+r, \eta(\varphi)\right)$, then $x_{t}\left(t_{0}, \varphi\right) \in \operatorname{Int} C_{+}$.

(A3) $f(t, \widehat{0})=f\left(t, \widehat{x^{*}}\right) \equiv 0$, where $x^{*} \triangleq\left(x_{1}^{*}, x_{2}^{*}, \cdots, x_{n}^{*}\right) \in \operatorname{Int} R_{+}^{n}, t \in R^{1}$.

(A4) For some $i$ and $\varphi \in C_{+} \backslash\left\{x^{*}\right\}$ such that $\varphi_{i}(0)-x_{i}^{*} \geq\left\|\varphi-x^{*}\right\|$, we have $f_{i}(t, \varphi)<0$ for $t \in R^{1}$.

(A5) For some $i$ and $\varphi \in \operatorname{Int} C_{+} \backslash\left\{x^{*}\right\}$ such that $x_{i}^{*}-\varphi_{i}(0) \geq\left\|\varphi-x^{*}\right\|$, we have $f_{i}(t, \varphi)>0$ for $t \in R^{1}$.

Proposition 3.2. Let (A1) and (A4) hold. If $\varphi \in C_{+} \backslash\{0\}$, then the set of $\left\{x_{t}\left(t_{0} ; \varphi\right): t \in\left[t_{0}, \eta(\varphi)\right)\right\}$ is bounded and hence $\eta(\varphi)=+\infty$.

Proof. For $\varphi \in C_{+} \backslash\{0\}$, we claim that $\left\|x_{t}\left(t_{0}, \varphi\right)-x^{*}\right\| \leq\|\varphi\|+2\left\|x^{*}\right\|$ for all $t \in\left[t_{0}, \eta(\varphi)\right)$. Otherwise, there exists $t_{1} \in\left(t_{0}, \eta(\varphi)\right)$ such that

$$
\left\|x_{t_{1}}\left(t_{0}, \varphi\right)-x^{*}\right\|>\|\varphi\|+2\left\|x^{*}\right\| .
$$

Let

$$
t^{*}=\inf \left\{t: t \in\left[t_{0}, \eta(\varphi)\right),\left\|x_{t}\left(t_{0}, \varphi\right)-x^{*}\right\|>\|\varphi\|+2\left\|x^{*}\right\|\right\} .
$$

Then $t^{*} \in\left(t_{0}, t_{1}\right)$ and

$$
\|\varphi\|+2\left\|x^{*}\right\|=\left\|x_{t^{*}}\left(t_{0}, \varphi\right)-x^{*}\right\| \geq\left\|x_{t}\left(t_{0}, \varphi\right)-x^{*}\right\| \text { for all } t \in\left[t_{0}, t^{*}\right] .
$$

In view of $(3.5)$ and $x_{t}\left(t_{0}, \varphi\right) \in C_{+}$, there exist $t^{* *} \in\left[t_{0}, t^{*}\right]$ and $i \in I$ such that

$$
x_{i}\left(t^{* *} ; t_{0}, \varphi\right)-x_{i}^{*}=\left|x_{i}\left(t^{* *} ; t_{0}, \varphi\right)-x_{i}^{*}\right|=\|\varphi\|+2|| x^{*}\|\geq\| x_{t^{* *}}\left(t_{0}, \varphi\right)-x^{*} \| .
$$

By (A4), we have $f_{i}\left(t^{* *}, x_{t^{* *}}\left(t_{0}, \varphi\right)\right)<0$. On the other hand, it follows from system (1.1) and the choice of $t^{* *}$ that $f_{i}\left(t^{* *}, x_{t^{* *}}\left(t_{0}, \varphi\right)\right)=x_{i}^{\prime}\left(t^{* *} ; t_{0}, \varphi\right) \geq 0$, a contradiction. This implies that the claim holds. Thus,

$$
\left\|x_{t}\left(t_{0}, \varphi\right)\right\| \leq\left\|x_{t}\left(t_{0}, \varphi\right)-x^{*}\right\|+\left\|x^{*}\right\| \leq\|\varphi\|+3\left\|x^{*}\right\|, \text { for all } t \in\left[t_{0}, \eta(\varphi)\right) .
$$

Finally, according to Theorem 3.1 in [4, p. 45], we easily obtain $\eta(\varphi)=+\infty$.

Theorem 3.1. Let (A1)-(A5) hold. Then $x^{*}$ is a globally stable equilibrium on $C_{+} \backslash\{0\}$.

Proof. Define $V: C_{+} \rightarrow C_{+}$such that $V(\varphi)=\left\|\varphi-x^{*}\right\| \triangleq \sup \left\{\left\|\varphi_{i}(\theta)-x_{i}^{*}\right\|: i \in\right.$ $I$ and $\left.\theta \in\left[-r_{i}, 0\right]\right\}$. Given $\varphi \in C_{+} \backslash\{0\}$, obviously, by (A2), we have

$$
x_{t}\left(t_{0}, \varphi\right) \in \operatorname{Int} C_{+} \text {for all } t \geq t_{0}+r .
$$

We next show that the following claims are true:

Claim (i). If there exists $T_{0} \geq t_{0}$ such that $x_{T_{0}}\left(t_{0}, \varphi\right)=x^{*}$, then $x_{t}\left(t_{0}, \varphi\right)=x^{*}$ for all $t \geq T_{0}$.

Claim (ii). If $x_{t}\left(t_{0}, \varphi\right) \neq x^{*}$ for all $t \in\left[t_{0},+\infty\right)$, then $V\left(x_{t}\left(t_{0}, \varphi\right)\right)$ is strictly decreasing on $\left[t_{0}+r,+\infty\right)$.

Claim (iii). $V\left(x_{t}\left(t_{0}, \varphi\right)\right)$ is non-increasing on $\left[t_{0}+r,+\infty\right)$. 
(i) Obviously, Claim (i) follows from (A3).

(ii) If Claim (ii) does not hold, there exist $t_{1}, t_{2} \in\left[r+t_{0},+\infty\right)$ such that $t_{1}<t_{2}$ and $V\left(x_{t_{2}}\left(t_{0}, \varphi\right)\right) \geq V\left(x_{t_{1}}\left(t_{0}, \varphi\right)\right)$. By the choice of $t_{1}$ and $t_{2}$, we can deduce that there exists $t_{3} \in\left[t_{1}, t_{2}\right]$ such that $\left\|x\left(t_{3} ; t_{0}, \varphi\right)-x^{*}\right\|=V\left(x_{t_{3}}\left(t_{0}, \varphi\right)\right)$. Thus, there exists $i \in I$ such that either $x_{i}\left(t_{3} ; t_{0}, \varphi\right)-x_{i}^{*}=V\left(x_{t_{3}}\left(t_{0}, \varphi\right)\right)$ or $x_{i}\left(t_{3} ; t_{0}, \varphi\right)-x_{i}^{*}=$ $-V\left(x_{t_{3}}\left(t_{0}, \varphi\right)\right)$. Note that $x_{t_{3}}\left(t_{0}, \varphi\right) \in \operatorname{Int}\left(C_{+}\right) \backslash\left\{x^{*}\right\}$. If the former holds, then by (A4) we have $f_{i}\left(t_{3}, x_{t_{3}}\left(t_{0}, \varphi\right)\right)<0$. On the other hand, it follows from (1.1) that $f_{i}\left(t_{3}, x_{t_{3}}\left(t_{0}, \varphi\right)\right)=x_{i}^{\prime}\left(t_{3} ; t_{0}, \varphi\right) \geq 0$, a contradiction. If the latter holds, then by (A5), (1.1) and a similar discussion, we can deduce a contradiction. Therefore, Claim (ii) follows.

(iii) Obviously, Claims (i) and (ii) imply Claim (iii).

Therefore, Theorem 3.1 follows from Proposition 3.1.

Theorem 3.1 is not completely satisfactory since it does not characterize the global stability of (1.1) solely in terms of properties of $f$. In the following, we give sufficient conditions on $f$ to guarantee assumptions (A1) and (A2).

(A11) There exist $k=\left(k_{1}, k_{2}, \cdots, k_{n}\right) \in R_{+}^{n}$ and a non-negative map $g: R^{1} \times$ $C_{+} \rightarrow R_{+}^{n}$ such that for all $\varphi \in C_{+}, f(t, \varphi)=-\operatorname{diag}\left(k_{1}, k_{2}, \cdots, k_{n}\right) \varphi(0)+g(t, \varphi)$.

(A12) For each $\varphi \in C_{+}$, denote $\bar{I}=\left\{i: \varphi_{i}\left(\theta_{i}\right)=0\right.$ for all $\left.\theta_{i} \in\left[-r_{i}, 0\right], i \in I\right\}$ and $\bar{J}=\left\{i: \varphi_{i}\left(\theta_{i}\right)>0\right.$ for all $\left.\theta_{i} \in\left[-r_{i}, 0\right], i \in I\right\}$. If $\bar{I} \neq \emptyset, \bar{J} \neq \emptyset$ and $\bar{I}^{\sharp}+\bar{J}^{\sharp}=n$, then there exists $i \in \bar{I}$ such that $f_{i}(t, \varphi)>0$ for all $t \in R^{1}$.

Lemma 3.1. Let (A11), (A12) and (A4) hold. Then for any $\varphi \in C_{+}$, we have either $x_{t}\left(t_{0}, \varphi\right)=0$ for all $t \geq t_{0}$ or $x_{t}\left(t_{0}, \varphi\right) \in$ Int $C_{+}$for all $t \geq t_{0}+(n+2) r$.

Proof. For $\varphi \in C_{+}$, using the assumption (A11) and Theorem 5.2.1 in [6, p. 81], we have $x_{t}\left(t_{0}, \varphi\right) \in C_{+}$for all $t \in\left[t_{0}, \eta(\varphi)\right)$, which, together with (A4), implies that the conclusion of Proposition 3.2 holds. Moreover, if $\varphi_{i}(0)>0$ for some $i$, then $x_{i}\left(t ; t_{0}, \varphi\right)>0$ for all $t \in\left[t_{0},+\infty\right)$. Hence for any $i \in I$, either $x_{i}\left(t ; t_{0}, \varphi\right)>0$ for all $t \geq t_{0}+r$ or $x_{i}\left(t ; t_{0}, \varphi\right)=0$ for all $t \in\left[t_{0}, t_{0}+r\right]$. Assume, by way of a contradiction, that the conclusion of Lemma 3.1 does not hold. Then there exists $t_{1} \in\left[t_{0}, t_{0}+r\right]$ such that $x_{i}\left(t_{1} ; t_{0}, \varphi\right)>0$ for some $i$.

Let $M_{t}=\left\{i \in I: x_{i}\left(t ; t_{0}, \varphi\right)>0\right\}$, where $t \geq t_{0}$. It follows that $M_{t_{1}} \neq \phi$ and $M_{s} \subseteq M_{t}$ when $t_{0} \leq s \leq t$.

Claim. If $t^{*} \in R_{+}^{1}$ and $M_{t^{*}} \notin\{\phi, I\}$, then $M_{t^{*}} \neq M_{t^{*}+r}$.

If the claim is not true, then $M_{t}=M_{t^{*}}, t \in\left[t^{*}, t^{*}+r\right]$. Thus, it follows from (A12) that there exists $i \in I \backslash M_{t^{*}+r}$ such that $f_{i}\left(t^{*}+r, x_{t^{*}+r}\left(t_{0}, \varphi\right)\right)>0$. Hence, from (1.1), we obtain

$$
x_{i}{ }^{\prime}\left(t^{*}+r ; t_{0}, \varphi\right)=f_{i}\left(t^{*}+r, x_{t^{*}+r}\left(t_{0}, \varphi\right)\right)>0 .
$$

Thus, there exists $\varepsilon>0$ such that

$$
\frac{\mathrm{d}\left(x_{i}\left(t ; t_{0}, \varphi\right)\right)}{\mathrm{d} t}>0, \quad t \in\left[t^{*}+r-\varepsilon, t^{*}+r\right] .
$$

Since $x_{t}\left(t_{0}, \varphi\right) \geq 0, \quad t \geq t_{0}$, we have $x_{i}\left(t^{*}+r ; t_{0}, \varphi\right)>0$. So, it follows that $i \in M_{t^{*}+r}$, which yields a contradiction. This completes the proof of the claim.

Now, we will show that $M_{t_{1}+(n-1) r}=I$. Otherwise, by the above claim, we have

$$
\phi \neq M_{t_{1}} \subseteq M_{t_{1}+r} \subseteq \ldots \subseteq M_{t_{1}+(n-1) r} \subseteq M_{t_{1}+n r},
$$

and $M_{t_{1}+i r} \neq M_{t_{1}+(i-1) r}, i \in I$. But this contradicts $M_{t} \subseteq I$. This completes the proof of Lemma 3.1. 
Remark 3.1. For $\varphi \in\left\{\varphi \in C_{+}: \varphi(0)>0\right\}$, using the proof used in Lemma 3.1, we have that $x_{t}\left(t_{0} ; \varphi\right) \in \operatorname{Int} C_{+}$for all $t \geq t_{0}+(n+2) r$.

Theorem 3.2. Let (A11), (A12) and (A3)-(A5) hold. Then $x^{*}$ is a globally stable equilibrium in the set of $\left\{\varphi \in C_{+}: \varphi(0)>0\right\}$.

Proof. Suppose $\varphi \in C_{+}$with $\varphi(0)>0$. Then by Remark 3.1, we have $x_{t}\left(t_{0}, \varphi\right) \in$ Int $C_{+}$for all $t \geq(n+2) r+t_{0}$. Define $V: C_{+} \rightarrow C_{+}$such that $V(\varphi)=\| \varphi-$ $x^{*} \| \triangleq \sup \left\{\left\|\varphi_{i}(\theta)-x_{i}^{*}\right\|: i \in I\right.$ and $\left.\theta \in\left[-r_{i}, 0\right]\right\}$. Using a similar argument as in Theorem 3.1, we may deduce that the following statements are true:

(i) If there exists $T_{0} \geq t_{0}$ such that $x_{T_{0}}\left(t_{0}, \varphi\right)=x^{*}$, then $x_{t}\left(t_{0}, \varphi\right)=x^{*}$ for all $t \geq T_{0}$.

(ii) If $x_{t}\left(t_{0}, \varphi\right) \neq x^{*}$ for all $t \in R_{+}^{1}$, then $V\left(x_{t}\left(t_{0}, \varphi\right)\right)$ is strictly decreasing on $\left[t_{0}+(n+2) r,+\infty\right)$.

(iii) $V\left(x_{t}\left(t_{0}, \varphi\right)\right)$ is non-increasing on $\left[t_{0}+(n+2) r,+\infty\right)$.

Consequently, Theorem 3.2 follows from Proposition 3.1. This completes the proof of Theorem 3.2.

Next, consider the following systems of delay differential equations:

$$
x_{i}^{\prime}(t)=f_{i}\left(t, x_{t}\right), f_{i}\left(t, x_{t}\right)=L_{i} x_{t}+x_{i}(t) g_{i}\left(t, x_{t}\right), i \in I,
$$

where $\bar{L} \triangleq\left(L_{1}, L_{2}, \cdots, L_{n}\right): C_{+} \rightarrow R^{n}$ is a bounded linear operator and $g \triangleq$ $\left(g_{1}, g_{2}, \cdots, g_{n}\right): R^{1} \times C_{+} \rightarrow R^{n}$.

Here, we give the following assumptions:

(C1) If $\varphi \in C_{+}$and $\varphi_{i}(0)=0$ for some $i$, then $L_{i}(\varphi) \geq 0$.

(C2) Denote $f(t, \varphi)=\bar{L} \varphi+\operatorname{diag} \varphi(0) g(t, \varphi)$ for all $\varphi \in C_{+}$. There exists $x^{*} \in$ Int $R_{+}^{n}$ such that $f\left(t, x^{*}\right)=0$ and $f$ satisfies assumptions (A4) and (A5).

(C3) $f$ satisfies assumption (A12).

In view of $(\mathrm{C} 1)$, it follows from Proposition 1.2 of [6] that system (3.6) satisfies condition (A1). Moreover, together with (A12), we can show that Lemma 3.1 also holds. Hence, by using a similar argument as in the proof of Theorem 3.2, we obtain the following result.

Theorem 3.3. Let $(\mathrm{C} 1)-(\mathrm{C} 3)$ hold. Then $x^{*}$ is a global stability equilibrium in the set of $\left\{\varphi \in C_{+}: \varphi(0)>0\right\}$.

\section{Applications}

In this section, we give several examples to illustrate the applications of the main results in Section 2.

Example 4.1. Consider the following non-autonomous Nicholson blowflies models with multi-patches:

$$
x_{i}^{\prime}(t)=\sum_{j=1}^{n} a_{i j} x_{j}(t)+\beta x_{i}\left(t-\tau_{i}(t)\right) e^{-x_{i}\left(t-\tau_{i}(t)\right)}-d x_{i}(t), \text { for } i=1,2, \cdots, n,
$$

where $\tau_{i}(t): R^{1} \rightarrow(0,+\infty)$ is almost periodic, $\beta>0, A=\left(a_{i j}\right)_{n \times n}$ is a cooperative and irreducible matrix, $r_{i}=\sup _{t \in R^{1}} \tau_{i}(t)$ and $\left(r_{1}, r_{2}, \cdots, r_{n}\right) \in \operatorname{Int} R_{+}^{n}$. In what follows, we always assume that $\frac{\beta}{d} \in\left[e, e^{2}\right]$ and $\sum_{j \neq i} a_{i j}=-a_{i i}$ for all $i$. 
Lemma 4.1 ([8, Lemma 2.3]). Let $\bar{\beta}=\frac{\beta}{d}$. If $a \geq 0$ and $b \geq 0$, then we have the following results:

(i) If $a-\ln \bar{\beta} \geq|b-\ln \bar{\beta}|$, then $-a+\bar{\beta} b e^{-b} \leq 0$. Moreover, $-a+\bar{\beta} b e^{-b}=0$ if and only if $a=b=\ln \bar{\beta}$.

(ii) If $\ln \bar{\beta}-a \geq|b-\ln \bar{\beta}|$, then $-a+\bar{\beta} b e^{-b} \geq 0$. Moreover, $-a+\bar{\beta} b e^{-b}=0$ if and only if either $a=b=\ln \bar{\beta}$ or $a=b=0$.

In Example 4.1, let $x^{*}=\left(\ln \frac{\beta}{d}, \ln \frac{\beta}{d}, \cdots, \ln \frac{\beta}{d}\right), D=\operatorname{diag}\{d, d, \cdots, d\}$ and $f(t, \varphi)$ $=A \varphi(0)-D \varphi(0)+\beta h(t, \varphi)$, where

$$
\begin{aligned}
& h(t, \varphi)=\operatorname{diag}\left\{\varphi_{1}\left(-\tau_{1}(t)\right) e^{\varphi_{1}\left(-\tau_{1}(t)\right)}, \varphi_{2}\left(-\tau_{2}(t)\right) e^{\varphi_{2}\left(-\tau_{2}(t)\right)},\right. \cdots, \\
&\left.\varphi_{n}\left(-\tau_{n}(t)\right) e^{\varphi_{n}\left(-\tau_{n}(t)\right)}\right\} .
\end{aligned}
$$

Remark 4.1. (i) For some $i$ and $\varphi \in C_{+} \backslash\left\{x^{*}\right\}$ such that $\varphi_{i}(0)-x_{i}^{*} \geq\left\|\varphi-x^{*}\right\|$, by Lemma 4.1 (i), we have

$$
\begin{aligned}
f_{i}(t, \varphi) & =\sum_{j=1}^{n} a_{i j} \varphi_{j}(0)+\beta \varphi_{i}\left(-\tau_{i}(t)\right) e^{-\varphi_{i}\left(-\tau_{i}(t)\right)}-\varphi_{i}(0) \\
& \leq \sum_{j=1}^{n} a_{i j} \varphi_{i}(0)+d\left(-\varphi_{i}(0)+\frac{\beta}{d} \varphi_{i}\left(-\tau_{i}(t)\right) e^{-\varphi_{i}\left(-\tau_{i}(t)\right)}\right)<0, t \in R^{1} .
\end{aligned}
$$

(ii) For some $i$ and $\varphi \in \operatorname{Int} C_{+} \backslash\left\{x^{*}\right\}$ such that $x_{i}^{*}-\varphi_{i}(0) \geq\left\|\varphi-x^{*}\right\|$, by Lemma 4.1 (ii), we obtain

$$
\begin{aligned}
f_{i}(t, \varphi) & =\sum_{j=1}^{n} a_{i j} \varphi_{j}(0)+\beta \varphi_{i}\left(-\tau_{i}(t)\right) e^{-\varphi_{i}\left(-\tau_{i}(t)\right)}-\varphi_{i}(0) \\
& \geq \sum_{j=1}^{n} a_{i j} \varphi_{i}(0)+d\left(-\varphi_{i}(0)+\frac{\beta}{d} \varphi_{i}\left(-\tau_{i}(t)\right) e^{-\varphi_{i}\left(-\tau_{i}(t)\right)}\right)>0, t \in R^{1} .
\end{aligned}
$$

Theorem 4.1. For system (4.1), if $\varphi \in C_{+}$with $\varphi(0)>0$, then

$$
\lim _{t \rightarrow \infty} x\left(t ; t_{0}, \varphi\right)=\left(\ln \frac{\beta}{d}, \ln \frac{\beta}{d}, \cdots, \ln \frac{\beta}{d}\right) .
$$

Proof. Since $A=\left(a_{i j}\right)_{n \times n}$ is a cooperative and irreducible matrix, by Lemma 4.1 and Remark 4.1, the assumptions (A11), (A12) and (A3)-(A5) hold. Consequently, Theorem 4.1 follows from Theorem 3.2.

Example 4.2. Consider the following non-autonomous delay differential systems: (4.3)

$x_{i}^{\prime}(t)=\sum_{j=1}^{n} a_{i j} x_{j}(t)+k_{i} x_{i}(t)\left[\alpha_{i}-b_{i 0} x_{i}(t)-\sum_{j=1}^{m} b_{i j} x_{i}\left(t-\tau_{j}(t)\right)\right]$, for $i=1,2, \cdots, n$,

where $\tau_{j}(t): R^{1} \rightarrow(0,+\infty)$ is almost periodic, $k_{i}>0, \alpha_{i}>0$ and $b_{i j} \leq 0, j=$ $1,2, \cdots, m . A=\left(a_{i j}\right)_{n \times n}$ is a cooperative and irreducible matrix, $r_{i}=\sup _{t \in R^{1}} \tau_{i}(t)$ and $\left(r_{1}, r_{2}, \cdots, r_{n}\right) \in \operatorname{Int} R_{+}^{n}$.

In this example, denote $b_{i}=\sum_{j=0}^{m} b_{i j}>0$, and $\alpha_{i}^{*}=\frac{\alpha_{i}}{b_{i}}, x^{*}=\left(\alpha_{1}^{*}, \alpha_{2}^{*}, \cdots, \alpha_{n}^{*}\right)$. Also, denote $f(t, \varphi)=\left(f_{1}(t, \varphi), f_{2}(t, \varphi), \cdots, f_{n}(t, \varphi)\right)$, where

$$
f_{i}(t, \varphi)=\sum_{j=1}^{n} a_{i j} \varphi_{j}(0)+k_{i} \varphi_{i}(0)\left[\alpha_{i}-b_{i 0} \varphi_{i}(0)-\sum_{j=1}^{m} b_{i j} \varphi_{i}\left(-\tau_{j}(t)\right)\right] \text { for } \varphi \in C_{+} .
$$


Lemma 4.2. If $\alpha_{i}^{*}=\alpha_{1}^{*}$ and $\sum_{j \neq i} a_{i j}=-a_{i i}$ for all $i \in I$, then $f$ satisfies assumptions (A3)-(A5) of Section 3.

Proof. Obviously, $x^{*} \in \operatorname{Int}\left(R_{+}^{n}\right)$ and $f\left(t, x^{*}\right)=0$; that is, (A3) follows. Let $\varphi_{i}(0)-$ $x_{i}^{*} \geq\left\|\varphi-x^{*}\right\|$ for some $i$ and $\varphi \in C_{+} \backslash\left\{x^{*}\right\}$. Then we have $\varphi_{i}(0)>x_{i}^{*}$ and

$$
\begin{aligned}
f_{i}(t, \varphi) & =\sum_{j=1}^{n} a_{i j} \varphi_{j}(0)+k_{i} \varphi_{i}(0)\left[\alpha_{i}-b_{i 0} \varphi_{i}(0)-\sum_{j=1}^{m} b_{i j} \varphi_{i}\left(-\tau_{j}(t)\right)\right] \\
& \leq \sum_{j=1}^{n} a_{i j} \varphi_{i}(0)+k_{i} \varphi_{i}(0)\left[\alpha_{i}-b_{i 0} \varphi_{i}(0)-\sum_{j=1}^{m} b_{i j} \varphi_{i}\left(-\tau_{j}(t)\right)\right] \\
& \leq \sum_{j=1}^{n} a_{i j} \varphi_{i}(0)+k_{i} \varphi_{i}(0)\left[\alpha_{i}-b_{i 0} \varphi_{i}(0)-\sum_{j=1}^{m} b_{i j} \varphi_{i}(0)\right] \\
& \leq k_{i} \varphi_{i}(0)\left[\alpha_{i}-b_{i 0} \varphi_{i}(0)-\sum_{j=1}^{m} b_{i j} \varphi_{i}(0)\right] \\
& \leq-k_{i} \varphi_{i}(0)\left(b_{i 0}+\sum_{j=1}^{m} b_{i j}\right)\left(\varphi_{i}(0)-\alpha_{i}^{*}\right) \\
& <0
\end{aligned}
$$

where $t \geq 0$. Thus, (A4) holds. By a similar argument as above, we can deduce that (A5) follows. This completes the proof.

In what follows, we always assume that $\alpha_{i}^{*}=\alpha_{1}^{*}$ and $\sum_{j \neq i} a_{i j}=-a_{i i}$ for all $i \in I$.

Lemma 4.3. If $\varphi \in C_{+}$, then $x_{t}\left(t_{0}, \varphi\right)$ exists and is unique on $\left[t_{0},+\infty\right)$.

Proof. Suppose $\varphi \in C_{+}$, Obviously, from Proposition 1.2 of [6], we obtain that $x_{t}\left(t_{0}, \varphi\right) \in C_{+}$is unique for $t \in\left[t_{0}, \eta\right)$, where $\left[t_{0}, \eta\right)$ is the maximal right-interval of existence of $x\left(t ; t_{0}, \varphi\right)$. We next show $\eta=\infty$. Denote $M_{t}=\sup \left\{\mid x_{i}\left(t+\theta ; t_{0}, \varphi\right)-\right.$ $x_{i}^{*} \mid: i \in I$ and $\left.\theta \in\left[t_{0}-r_{i}, t_{0}\right]\right\}$ for all $t \in\left[t_{0}, \eta\right)$. We now claim $M_{t}$ is non-increasing on $\left[t_{0}, \eta\right)$. Otherwise, without loss of generality, we may assume that there exists $i \in I$ and $t^{*} \geq t_{0}$ such that $x_{i}\left(t^{*} ; t_{0}, \varphi\right)-\alpha_{i}^{*}>\sup \left\{\left|x_{i}\left(t ; t_{0}, \varphi\right)-x_{i}^{*}\right|: i \in I\right.$ and $t \in$ $\left.\left[t^{*}-r_{i}, t^{*}\right)\right\}$ and hence $x_{i}^{\prime}\left(t^{*} ; t_{0}, \varphi\right) \geq 0$. On the other hand, it follows from (4.3) that

$$
\begin{aligned}
x_{i}^{\prime}\left(t^{*} ; t_{0}, \varphi\right)= & \sum_{j=1}^{n} a_{i j} x_{j}\left(t^{*} ; t_{0}, \varphi\right)+k_{i} x_{i}\left(t^{*} ; t_{0}, \varphi\right)\left[\alpha_{i}-b_{i 0} x_{i}\left(t^{*} ; t_{0}, \varphi\right)\right. \\
& \left.-\sum_{j=1}^{m} b_{i j} x_{i}\left(t^{*}-\tau_{j}\left(t^{*}\right) ; t_{0}, \varphi\right)\right] \\
\leq & k_{i} x_{i}\left(t^{*} ; t_{0}, \varphi\right)\left[\alpha_{i}-b_{i 0} x_{i}\left(t^{*} ; t_{0}, \varphi\right)-\sum_{j=1}^{m} b_{i j} x_{i}\left(t^{*}-\tau_{j}\left(t^{*}\right) ; t_{0}, \varphi\right)\right] \\
\leq & -k_{i} x_{i}\left(t^{*} ; t_{0}, \varphi\right)\left(b_{i 0}+\sum_{j=1}^{m} b_{i j}\right)\left(x_{i}\left(t^{*} ; t_{0}, \varphi\right)-\alpha_{i}^{*}\right) \\
< & 0 .
\end{aligned}
$$

This yields a contradiction, and hence the claim follows. Consequently, $M_{t} \leq M_{0}$ for all $t \in\left[t_{0}, \eta\right)$ and thus $x_{i}\left(t, t_{0}, \varphi\right) \leq M_{0}+\alpha^{*}$ for all $t \in\left[t_{0}, \eta\right)$. Therefore, by Theorem 3.1 in [2, p. 45], $\eta=\infty$. This completes the proof. 
Theorem 4.2. For system (4.3), if $\varphi \in C_{+} \backslash\{0\}$, then $\lim _{t \rightarrow \infty} x\left(t ; t_{0}, \varphi\right)=$ $\left(\alpha_{1}^{*}, \alpha_{2}^{*}, \cdots, \alpha_{n}^{*}\right)$.

Proof. Since $A=\left(a_{i j}\right)_{n \times n}$ is a cooperative and irreducible matrix, by Lemmas 4.2 and 4.3 , the assumptions $(\mathrm{C} 1)-(\mathrm{C} 3)$ hold. Consequently, Theorem 4.2 follows from Theorem 3.3.

\section{REFERENCES}

1. K. Gopalsamy, Stability and Oscillation in Delay Differential Equations of Population Dynamics. Kluwer Academic Publishers, Dordrecht, 1992. MR.1163190 (93c:34150)

2. K. Hale, S.M. Verduyn Lunel, Introduction to Functional Differential Equations, SpringerVerlag, New York, 1993. MR.1243878 (94m:34169)

3. O. Ignatyev, On the asymptotic stability in functional differential equations, Proceedings of the American Mathematical Society, 127(6) (1999), 1753-1760. MR.1636954 (99i:34108)

4. S.H. Saker, Oscillation of continuous and discrete diffusive delay Nicholson's blowflies models, Applied Mathematics and Computation, 167 (2005), 179-197. MR.2170588 (2006e:34145)

5. H.L. Smith, Monotone Dynamical Systems, Math. Surveys Monogr., Amer. Math. Soc., Providence, RI, 1995. MR:1319817 (96c:34002)

6. H.L. Smith, Monotone semiflows generated by functional differential equations, J. Differential Equations, 66 (1987), 420-442. MR876806 (88j:34155)

7. J.W.-H. So, J.S. Yu, Global stability for a general population model with time delays, in: S. Ruan et al. (eds.), Differential Equations with Applications to Biology, Fields Institute Communications, vol. 21, American Mathematical Society, Providence, RI, 1999, pp. 447-457. MR1662633 (99m:92039)

8. T. S. Yi and X. Zou, Global attractivity of the diffusive Nicholson blowflies equation with Neumann boundary condition: A non-monotone case, J. Differential Equations, 245(11) (2008), 3376-3388. MR 2460028

9. T. S. Yi and L. H. Huang, Convergence for pseudo monotone semiflows on product ordered topological spaces, J. Differential Equations, 214 (2005), 429-456. MR2145256 (2006a:37017)

College of Mathematics and Information Engineering, Jiaxing University, Jiaxing, Zhejiang 314001, People's Republic of China

E-mail address: liubw007@yahoo.com.cn 